\title{
Solidarität ist das humanistische Fundament der Arbeitswelt
}

KAI LINDEMANN

\section{Solidarität als gewerkschaftlicher Grundwert}

Das Prinzip Solidarität ist die DNA der Gewerkschaften. Sie ist ihr Geschäftsmodell. Zählte man das Wort unter den Mai-Motti des DGB, würde es vermutlich an der Spitze liegen. Interessenpolitisch geht es in der Arbeitswelt nur gemeinsam, ob im Kampf für höhere Löhne oder in der Unterstützung kranker, alter, behinderter oder junger Kolleginnen und Kollegen. Das schafft Gemeinschaft, Kultur und Identität. Und das unterscheidet gewerkschaftliche Solidarität von anderen Definitionen und Deutungen solidarischer Muster wie der christlichen Nächstenliebe, der autokratischen Kameradschaft oder der bürgerlichen Philanthropie.

Gewerkschaftliche Solidarität kommt im Kollektiv zur Geltung. Sie begründet die kollektive Identität der Kolleginnen und Kollegen trotz aller individuellen, selbst politischen Unterschiede. Deshalb ist das Prinzip der Einheitsgewerkschaft für uns in Deutschland eine so wichtige Grundlage für Solidarität: Weil nicht nur in der Tarifpolitik Zusammenhalt gefordert ist, auch gegen politische Gefahren können sich die Gewerkschaften nur als Einheit wirksam wehren. Das ist die Lehre aus dem Faschismus. Solidarität wurde dadurch zu einem universellen Grundwert der Gewerkschaften.

Zwei Dimensionen hat die solidarische Politik der Gewerkschaften:

(1) Zum ersten handelt es sich um die organisations- und tarifpolitische Dimension, die sich auf Betriebe und Branchen konzentriert. Mit ihr werden die demokratischen Voraussetzungen für gelebte Solidarität geschaffen, mit Betriebsräten und der Bindung des Betriebs an aktuelle Tarifabschlüsse. Zwangsläufig greifen hier die unterschiedlichen Identitätsbezüge der Beschäftigten - Betrieb, Branche, Beruf - ineinander. Die kollektive Identität der Einheitsgewerkschaften hält sie zusammen. Solidarität ist die entscheidende Machtressource der abhängig Beschäftigten, die in der tagtäglichen Arbeit von Betriebsräten und Vertrauensleuten wirkt. Deshalb sind die Grenzen der gewerkschaftlichen Solidarität auch die Grenzen der gewerkschaftlichen Macht.

(2) Die zweite Dimension gewerkschaftlicher Solidarität resultiert aus ihrer Entstehungsgeschichte durch Hilfsund Unterstützungskassen. Es ist die soziale Sicherheit in einer solidarischen Gemeinschaft, die vom heutigen Sozialstaat erfüllt wird. Dabei geht es um den Anspruch, armen, kranken und hilfsbedürftigen Menschen die gesellschaftliche Teilhabe zu ermöglichen. Hierzu hat sich ein Sozialversicherungssystem entwickelt, das in seiner selbstverwalteten Struktur maßgeblich von den Gewerkschaften gestaltet wurde. Gesellschaftliche Solidarität ist das Fundament zur Stärkung der Selbstermächtigung hilfsbedürftiger Menschen. Zugleich definiert sie das Selbstverständnis der Gesellschaft über Humanität und soziale Würde.

An den beiden Dimensionen wird deutlich, wie eng gewerkschaftliche Solidarität an gesellschaftliche Gerechtigkeitsvorstellungen geknüpft ist, an Gerechtigkeit im Betrieb und an Gerechtigkeit in der Gesellschaft. Gerechtigkeit kann aber ebenso wenig wie Solidarität klar definiert werden. Beide Begriffe konkretisieren sich erst in der Praxis über die Beurteilung sozialer Verhältnisse.

Gegenwärtig ist viel vom Verlust des „gesellschaftlichen Zusammenhalts" die Rede. Hierfür sind auch materielle gesellschaftliche Entwicklungen verantwortlich. Armut hat sich in den westlichen Gesellschaften trotz guter Beschäftigungslage und konstantem Wirtschaftswachstum stark ausgebreitet. Vermögen und Einkommen sind immer ungleicher verteilt. Die Menschen empfinden wachsende Ungleichheiten nicht nur als unsolidarisch, sondern oft auch als Betrug, wodurch sie das Vertrauen in die gesellschaftlichen Institutionen verlieren. Ihre Gerechtigkeitserwartungen an die Politik sind enttäuscht. Denn viele Menschen haben einen „ungeschriebenen Gesellschaftsvertrag " ${ }^{\text {1 }}$ verinnerlicht, an dem sie die gesellschaftlichen Verhältnisse messen. 
Daran wird deutlich: Solidaritätsansprüche entstehen nicht primär aus sozialer Not und Elend, sondern aus gemeinsam empfundener Ungerechtigkeit und Willkür auch bezüglich anderer benachteiligter Gruppen. So ist Solidarität in ihrer praktischen, politischen Ausformulierung stets von „empfundener“ gesellschaftlicher oder betrieblicher Ungerechtigkeit abhängig. Damit resultiert sie immer aus Kritik und Empörung. Das stärkt den offenen demokratischen Diskurs.

Solidaritätsansprüche sind aber keinesfalls nur fortschrittlich, sie können sich auch reaktionär gegen Menschengruppen richten und auf das gefährlichste Kollektiv - „das Volk“ - fokussieren. Das ist dann exklusive Solidarität, die arme und bedürftige Personen als Betrüger an der Solidargemeinschaft diskriminiert. Diese Solidarität hat nichts mit Humanismus und den universellen Werten der Gewerkschaften zu tun. Sie macht es sich leicht, indem sie auf den einfachsten Ressentiments aufbaut und den alltäglich erlebten ökonomischen Wettbewerb auf alle zwischenmenschlichen Beziehungen projiziert.

Weil aber Gewerkschaften seit über 150 Jahren mit dem Geschäftsmodell Solidarität Erfolg haben, sind sie auch davon überzeugt, dass inklusive, humanistische Solidarität fortschrittlich ist und den wahren Interessen der Menschen entspricht. Soziale Empathie, Hilfsbereitschaft, Respekt, Altruismus und Toleranz sind Eigenschaften der menschlichen Natur, die sich weder durch einen radikalen Egoismus noch durch Ressentiments ersetzen lassen. Geben, teilen und gemeinsam für eine gute Sache erfolgreich kämpfen sind überzeugende Eigenschaften der solidarischen Gewerkschaftsbewegung.

Einige DGB-Gewerkschaften haben diesen Anspruch an Solidarität in ihren Satzungen aufgenommen. Die EVG und Ver.di, die IG BAU und die IG BCE bekennen sich ausdrücklich zu solidarischem Handeln und verpflichten ihre Mitglieder teilweise dazu. ${ }^{2}$ In den gewerkschaftlichen Grundsatzpapieren wird aber auch deutlich, wie unterschiedlich der Begriff gedeutet wird. Im betrieblichen Alltag wird eher von Kollegialität die Rede sein, und erst wenn sich Aktionen und Forderungen stärker politisieren und womöglich nach "Außen“ richten, ist die Rede von solidarischem Handeln. Und auch diese Deutungen sind von politischen Trends und Diskursen beeinträchtigt. Wo vor 100 Jahren von Solidarität die Rede war, könnte heute von sozialer Verantwortung oder gegenseitigem Respekt gesprochen werden.

\section{Solidarität im Wandel}

Allgemein kann Solidarität als eine Beziehungsform verstanden werden, die auf der gemeinsamen Identifikation über ein gemeinsames Problem basiert. In den heutigen marktliberalen Gesellschaften ist zwar die Problem-Identifikation gegeben, aber kollektive Beziehungsformen haben sich verändert. Robert Putnam beschrieb in seinem vielbeachteten Buch „Bowling Alone“ schon im Jahr 2000 den Niedergang kollektiver Beziehungsformen anhand der amerikanischen „Communities“. ${ }^{3}$ Auch in seiner Analyse wurde deutlich, dass der individuelle Anspruch an Selbstverwirklichung Solidarität verändert, erweitert und deren klassische Beziehungsmuster beeinträchtigt. Die heutige gesellschaftliche Fokussierung auf Marktindividuen impliziert eine gewisse Ignoranz gegenüber kollektiven Interessen, die Gewerkschaften schon oft verspürten.

Anfang des Jahrtausends war das öffentliche Ansehen der Gewerkschaften schlecht. Arbeitslosigkeit und geringes Wachstum ließen in der politischen Debatte keinen Platz für Solidarität - ganz im Gegenteil: Der Sozialstaat stand auf dem Prüfstand. Gewerkschaftliche Solidarität galt als Blockade grundlegender Reformen. Nach der Finanzkrise 2008 hat sich das Ansehen der Gewerkschaften verbessert, auch weil sie durch aktive Krisenpolitik ihre solidarische Kompetenz bewiesen. Sie wurden wieder stärker als notwendige Anwälte der arbeitenden Bevölkerung wahrgenommen. Marktliberalismus und Globalisierung hatten aber inzwischen die Arbeitsgesellschaft enorm verändert, die Folgen blieben und stellen auch heute noch die Gewerkschaften vor die Herausforderung, Solidarität zeitgemäßer zu formulieren und zu organisieren.

$\mathrm{Zu}$ diesen Herausforderungen zählt die mangelnde betriebliche Integration der Belegschaften. Die Arbeitssoziologie spricht von einer „Entgrenzung der Arbeit“ ${ }^{4}$, die sich an Leiharbeitskräften, neuen Selbstständigen - zumeist auf Werkvertragsbasis -, Minijobbern und anderen prekären Arbeitsformen festmacht. Sie spalten und hierarchisieren die Belegschaften, sodass es schwieriger wird, die solidarische Gemeinschaft zu organisieren. Auch das Subunternehmertum - primär in der Baubranche und Fleischindustrie - spaltet die Belegschaften in der Wertschöpfungskette; mit äußerst problematischen Folgen für den Arbeitsschutz, wie wir jüngst am Tönnies-Skandal sehen konnten. Wenn betriebliche Grenzen verschwimmen, behindert das solidarisches Handeln. Die Entgrenzung

1 Vgl. Moore, B. (1982): Ungerechtigkeit. Die sozialen Ursachen von Unterordnung und Widerstand, Frankfurt a. M.

2 Vgl. im Einzelnen die Satzungen der Eisenbahn- und Verkehrsgewerkschaft (https://www.evg-online.org/fileadmin/ UEber_uns/Satzung/19-12-05-evg_satzung_191113_DIN_ A4_WEB.pdf), der Vereinten Dienstleistungsgewerkschaft (https://wdr.verdi.de/ueber-uns/satzung-regelungen), der IG Bauen-Agrar-Umwelt (https://igbau.de/Die-Satzung.html) und der IG Bergbau, Chemie, Energie (https://m.igbce.de/ blob/8538/8b69d6gbff699012e19323f1b305d827/satzungdata.pdf) (letzter Zugriff jeweils: 11. 05. 2020).

3 Putnam, R. D. (2000): Bowling Alone. The Collapse and Revival of American Community, New York

4 Vgl. Ludwig, C./ Simon, H./ Wagner, A. (Hrsg.) (2019): Entgrenzte Arbeit, (un)begrenzte Solidarität? Bedingungen und Strategien gewerkschaftlichen Handelns im flexiblen Kapitalismus, Münster 
der Arbeit ist ein sozialer Prozess der Restrukturierung, also auch der partiellen Rückentwicklung zu ursprünglichen kapitalistischen Strukturen. Sie verursacht zugleich eine Entgrenzung der traditionellen gewerkschaftlichen Organisationsstrategien: Beschäftigte müssen mit verschiedenen Solidaritätsangeboten angesprochen werden, damit der Entgrenzung keine Entsolidarisierung folgt.

Eine weitere betriebspolitische Herausforderung ist die (oft mit Privatisierung verbundene) Ausgliederung vormals gut organisierter Betriebsteile. Auch in vielen neuen Geschäftsmodellen (z. B. Startups) ist die Entgrenzung der Arbeit von vornherein festgeschrieben. Insbesondere die digitale Gig-Economy der Plattformen verzichtet auf klare Betriebsstrukturen und versperrt so den Weg zur Organisierung der Beschäftigten nach klassischen Methoden. Hinzu kommt ein Wertewandel bei jungen Beschäftigten, die vermehrt andere Prioritäten in der solidarischen Praxis setzen. Sie wollen autonomer ihre Solidarität kundtun und betonen die Diversität im Kollektiv. Solidarität wird weniger als politische „Verpflichtung für das Ganze“ gesehen, sondern vielmehr als persönliche, moralische Haltung.

Viele der hier genannten Problemkontexte stehen bei den DGB-Gewerkschaften ganz weit oben auf der politischen Agenda und werden teilweise auch von der Politik aufgegriffen - insbesondere im Kontext der digitalen Arbeitswelt. Die gewerkschaftsfeindliche Wirtschaftswelt ruht aber nicht und ist in den letzten Jahren wieder aggressiver geworden. Arbeitgeber verschließen sich gegen Maßnahmen einer stärkeren Tarifbindung mit ihrer Mitgliedschaft in Arbeitgeberverbänden ohne Tarifbindung (OT-Mitgliedschaft). Sie beharren auf ihrer autoritären Deutungshoheit, z.B. bei Flexibilisierungsmaßnahmen, die vom Management entschieden werden und die Selbstbestimmung der Beschäftigten einschränken. Zudem zeigen seit einigen Jahren manche Unternehmer ihr wahres Gesicht, wenn sie mit Anwälten gegen Betriebsräte und Gewerkschaften und damit direkt gegen gewerkschaftliche Solidarität vorgehen. Sie haben sich das „Union Busting“ " in den USA abgeschaut - an nichts wird deutlicher, welche Gefahr Kollektive, Demokratie und Solidarität für den radikalen Marktliberalismus darstellen. Schon Pierre Bourdieu hat in den 1990er Jahren konstatiert, dass der Neoliberalismus ,ein Programm der planmäßigen Zerstörung der Kollektive" ${ }^{6}$ sei.

Die gegenwärtig von Arbeitgebern geforderte Modularisierung von Tarifverträgen und die damit einhergehende Unterminierung der Flächentarifverträge zielt auf die Entmachtung kollektiver Verhandlungsprozesse. Aber auch die Individualisierung sozialer Sicherheit mit dem Postulat der Eigenverantwortung entkräftet kollektive gesellschaftliche Solidarsysteme der Sozialversicherung. Hierzu passt auch die Rede von einer „Subjektivierung der Arbeit“, die Arbeitnehmer zu Unternehmern ihrer Arbeitskraft macht oder machen soll. Eine radikal-liberale Gesellschaft der vielen "freien Unternehmer" ist eine Illusion, die schon im nachrevolutionären England des 17. Jahrhunderts von den Levellers erprobt wurde. ${ }^{7}$ Trotzdem ist die Ideologie der "Arbeitskraftunternehmer" in der Arbeitswelt inzwischen verbreitet und wird gestützt von den Selbstbestimmungsansprüchen der Erwerbstätigen selbst. ${ }^{8}$ Sie ist aber eine Gefahr für solidarisches Handeln, wenn über die Vermarktlichung betrieblicher Abläufe (Profit Center) Belegschaften in Gewinner und Verlierer gespalten werden.

\section{Ansprüche an Solidarität sind Herausforderungen für die Gewerkschaften}

Neue Formen der Solidarität zeigen sich im Spannungsfeld von Selbstbestimmung und Mitbestimmung, Eigennutz und Altruismus - etwa wenn Solidarität stärker zur Mobilisierung für ein spezifisches politisches Interesse genutzt wird und die kollektive Identität der beteiligten Gruppen in den Hintergrund gerät. Fridays for Future, die Solidarisierungen mit Geflüchteten 2015, die \#Unteilbar-Demo 2018 sind nur einige Beispiele für diese neuen Solidaritätsformen. Doch auch in der Arbeitswelt gibt es Anzeichen und Beispiele für neue Spielarten der Solidarisierung. In neuen digitalen Branchen und Betrieben werden oft grenzüberschreitende Strategien der Belegschaftsorganisation erprobt. Aber auch in traditionell gewerkschaftlich organisierten Betrieben ändern sich Ansprüche an Solidarität. Jüngere Beschäftigte fordern häufig mehr Selbstbestimmung und Partizipation ein. Auch die vielen Initiativen für solidarisches Wirtschaften und die vermehrten Gründungen von Genossenschaften unterstreichen das aktuelle Interesse an Solidarität. Solidarisierungen sind aber heute spontaner als in der Vergangenheit. Sie binden sich öfter an ein spezifisches Interesse und stellen damit weniger die Rolle des Kollektivs in den Vordergrund. Darauf müssen sich die Gewerkschaften einstellen.

Die Konjunktur der Solidaritäts-Debatte spiegelt sich auch in vielen Publikationen der letzten Jahre, die auch in den DGB-Gewerkschaften diskutiert werden. ${ }^{9}$ Eines wird in den heutigen Zeiten rasanten gesellschaftlichen Wandels deutlich: Die Solidaritätspolitik der Gewerkschaften braucht eine stärker vorausschauende Perspekti-

5 Vgl. IG Metall: Was ist Union Busting?, https://igmetallmehr-werden.de/union-busting-was-ist-das (letzter Zugriff: 11. 05. 2020)

6 Bourdieu, P. (1998): Gegenfeuer. Wortmeldungen im Dienste des Widerstands gegen die neoliberale Invasion, Konstanz, S. 26

7 Vgl. Anderson, E. (2019): Private Regierung, Frankfurt a. M

8 Vgl. Pongratz, H. J./ Voß, G. G. (Hrsg.) (2004): Typisch Arbeitskraftunternehmer? Befunde der empirischen Arbeitsforschung, Berlin

9 Vgl. z. B. Bude, H. (2019): Solidarität. Die Zukunft einer großen Idee, München. 
ve. Es kann in einer dynamischen Marktwirtschaft nicht mehr allein um die Verteidigung des Bestehenden gehen. Ansonsten würde auch das Prinzip der Solidarität als ein veraltetes Prinzip Schaden erleiden. Ebenso wie sich die Wirtschaft, die Gesellschaft und die Arbeitsverhältnisse unter den Bedingungen der ökologischen und digitalen Transformation verändern, müssen auch Gewerkschaften Solidarität für die Arbeitswelt neu begründen. Dabei geht es nicht um ein passives Sich-Anpassen, sondern darum, den Kolleginnen und Kollegen ein positives und überzeugendes Zukunftskonzept zu bieten. Solidarität ist immer auch eine Vision, wie wir zusammen arbeiten und leben wollen, wie wir Arbeit in Zukunft gestalten wollen und vor allem wie wir gemeinsam unsere Machtressource nutzen für Selbstbestimmung und eine Stärkung der Demokratie in Betrieb und Gesellschaft.

Die Corona-Krise hat gleichermaßen gezeigt, wie wichtig Solidarität für unser Gemeinwesen ist, aber auch, wie wichtig Gewerkschaften als Treiber solidarischen Handelns in der Krise sind. Die DGB-Gewerkschaften haben Beachtliches erreicht, darunter die Aufstockung des Kurzarbeitergeldes und die Unterstützung für Eltern und Auszubildende; sie haben Arbeitsschutzmaßnahmen in diversen Betrieben und Branchen gestaltet und intensiv Einfluss auf die Konjunkturprogramme genommen. Diese Krise wird gesellschaftliche und gewerkschaftliche Solidarität weiter fördern.

Langfristig ist für Gewerkschaften Solidarität in erster Linie eine organisationspolitische Aufgabe, die im Betrieb beginnt und auf internationaler Ebene endet. Gewöhnlich geht man davon aus, dass die Intensität solidarischen Handelns von der untersten Ebene zur obersten Ebene schwächer wird, was bezüglich des persönlichen Erfahrungshorizonts naheliegend ist. In Zukunft könnte aber die Form der internationalen Solidarität eine größere Bedeutung erlangen, weil koordinierte Arbeitskämpfe in der internationalen Arbeitsteilung notwendiger werden.

Die letzten Arbeitskämpfe von Ver.di bei Amazon sind ebenfalls gute Beispiele für eine neue, internationale Strategie. Ein gelungener Ausdruck internationaler Solidarität war auch die Ver.di/CWA-Kampagne „We expect better", mit der ab 2011 gemeinsam Druck auf T-Mobile USA, die Tochter der deutschen Telekom, gemacht wurde. T-Mobile ist seit 2001 auf dem US-Markt engagiert und hat sich seitdem sehr gewerkschaftsfeindlich gezeigt. Die dortige Schwestergewerkschaft von Ver.di, die Communication Worker Association (CWA), hat um Unterstützung gebeten. Es fanden Unterschriften-Sammlungen, Protestaktionen in beiden Ländern, Veranstaltungen, betriebliche Aktionen und Partnerschaften statt und beide Gewerkschaftsdachverbände (DGB und AFL/CIO) haben die Kampagne massiv unterstützt. Auch die Aktionen der Hafenarbeiter aus der Europäischen Transportarbeiterföderation (ETF) sind vorbildliche Beispiele für internationale Solidarität. Sie entladen keine Schiffe, wenn dadurch Arbeitskämpfe an anderen Orten sabotiert werden können.
Ein tragendes Element der internationalen Solidarität sind die Europäischen Betriebsräte (EBR), die dort für Solidarität sorgen, wo Standorte gegeneinander ausgespielt werden sollen. Der EBR von General Motors (Opel) hat sich hier besonders hervorgetan. Er intervenierte in Umstrukturierungspläne für spezifische Standorte und erarbeitete konstruktive, solidarische Alternativen.

Zunehmend fordern Belegschaften und Betriebsräte die Solidarität von Politik und Gesellschaft für ihre Arbeitskämpfe ein. Der Streik der Beschäftigten der GildeBrauerei für einen Haustarifvertrag ist ein jüngeres Beispiel hierfür. Am 29. Februar 2020 haben DGB und Politik auf einer Demonstration in Hannover ihre Solidarität mit den Gilde-Kolleginnen und -Kollegen gezeigt. Ein weiteres öffentlichkeitswirksames Beispiel zeigten Beschäftigte im Siemens-Kompressorenwerk in Leipzig-Plagnitz. Sie haben zwei Jahre für den Erhalt ihres Werks gekämpft und konnten dank der solidarischen Unterstützung der Bevölkerung über eine Online-Petition mit 8900 Unterschriften dessen Schließung verhindern. In einem anderen Siemens-Werk (Energy Management) in Leipzig hat der Betriebsrat seine Solidarität mit Geflüchteten bewiesen und ein Integrationsprojekt mit der Betriebsführung und den Behörden angestoßen, über das Asylbewerber erfolgreich in den Arbeitsmarkt integriert werden. Derartige Projekte gab es auch in vielen anderen Unternehmen, zumeist von den Betriebsräten angestoßen und verhältnismäßig wenig in der lokalen Öffentlichkeit thematisiert. Die Medienöffentlichkeit ist der Schlüssel für den Erfolg gewerkschaftlicher Solidarität, denn darüber lassen sich Politik und Gesellschaft in die Verantwortung nehmen, und zugleich wird für die Kraft kollektiver Identität geworben.

An den Beispielen sehen wir, dass Gewerkschaften tagtäglich Solidarität organisieren, neue Formen testen und bereit sind, ihre traditionellen Prinzipien der kollektiven Identität mit neuartigen Solidaritätsansprüchen zu verbinden. Sie müssen sich dafür stärker im gesellschaftspolitischen Diskurs positionieren und Identifikationspotenziale anbieten. Hierfür sollten sie offener, transparenter und vielfältiger werden, um die Ansprüche aller Arbeitnehmerinnen und Arbeitnehmer zu integrieren. Die Grundvoraussetzungen der gewerkschaftlichen, humanistischen Solidarität sind nicht verschwunden. Wir müssen nur tagtäglich daran arbeiten, sie an den Interessen aller Arbeitnehmerinnen und Arbeitnehmer neu auszurichten, um eine wirkungsvolle Politik zu betreiben.

\section{AUTOR}

KAI LINDEMANN, Dr. phil., Referatsleiter in der Grundsatzabteilung des DGB Bundesvorstands. Arbeitsschwerpunkte: Wandel der Arbeitswelt (z. B. Digitalisierung, ökologischer Umbau), Innovationspolitik, Zukunft der Gewerkschaften.

kai.lindemann@dgb.de 